\title{
Hydrodynamical Models of Mixing beyond a Convection Zone
}

\author{
Bernd Freytag \\ Institute for Astronomy and Space Physics, Uppsala, Sweden
}

\begin{abstract}
Numerical radiation hydrodynamics simulations of stellar surface convection have gained a high level of reliability and perform very well in the confrontation with observations. Similar simulations of convection zones in stellar interiors are much more difficult to set up because of the huge range of time-scales to cover. Therefore, simplified models have to be used, where the results have to be carefully extrapolated to apply them to stellar interiors. Simulations of shallow surface convection zones show that the overshooting velocity fields extend far beyond the region with significant convective energy flux.
\end{abstract}

The description of convection has far-reaching consequences for the quality of the entire model of a stellar atmosphere or the internal structure. A particular burning question concerns the actual extent of a convection zone. Hitherto, in almost all cases only local 1D models are used to describe convection. These rely on the Schwarzschild criterion to specify the boundaries of a convection zone: motions are restricted to the convectively unstable layers.

On the other hand, spatially and temporally resolved observations of the solar photosphere reveal that these convectively stable layers are in fact full of convection induced flows (see e.g. Krieg et al. 2000). The fitting of observed spectra of many stars can only be achieved with 1D models including convective micro- and macroturbulent velocities (see e.g. Landstreet 1998).

The observational evidence of overshoot in stellar interiors is more indirect: The widening of the main-sequence in the region of the A-type stars is explained by core overshoot (Pols et al. 1997). On the other side, helioseismology still fails to find evidence for significant overshoot below the solar convection zone (Schlattl \& Weiss 1999; Christensen-Dalsgaard, Monteiro, \& Thompson 1995). Clearly, there is convective overshoot in stars. Yet, a convincing quantitative model of convection including overshoot is still missing (but see Kupka 1999).

Detailed 2D or 3D radiation hydrodynamics simulations of stellar surface convection are basically characterized by the same parameters as classical $1 \mathrm{D}$ atmospheres, i.e. effective temperature, gravity, and chemical composition (see e.g. Freytag, Ludwig, \& Steffen 1996, Stein \& Nordlund 1998). There are other detailed model specifications like numerical resolution, model extent, or artificial viscosity parameters. However, there is no tunable parameter as in the mixinglength theory, that would allow the easy adjustment of the convective flux.

Elements of such a code are checked with standard test problems. But the main challenge for every numerical simulation is the confrontation with observations. The Sun allows us to study the properties of its surface granulation with high spatial, temporal and/or spectral resolution. In a recent work, Asplund 
et al. (2000) investigate "The effects of numerical resolution on hydrodynamical surface convection simulations and spectral line formation". They find that, while 2D simulations give only qualitatively useful results, even 3D models with modest resolution already produce reliable temperature/pressure stratifications. And at the highest resolution $\left(200^{2} \times 82\right.$ points) the synthetical "line shapes have converged essentially perfectly to the observed profiles", which means that also the velocities are represented accurately.

The good agreement between the properties of simulated and observed granulation (Stein \& Nordlund 1998) as well as between theoretical and observed line profiles (Asplund et al. 2000) allows the conclusion that the numerical models are an excellent representation of real stellar surface convection. In addition, there is no hint that an even further increase of the numerical resolution would alter this picture, because the observed line profiles already contain information about all scales and the simulations at the present resolution already give almost perfectly matching profiles.

Table 1. Characteristic numbers at the surface (here the solar photosphere) and in the interior (bottom of the solar convection zone)

\begin{tabular}{c|cc}
\hline & Surface & Interior \\
\hline Mach number & 1 & 0.001 \\
turnover time/Courant time & 1000 & $10^{6}$ \\
thermal time/turnover time & 10 & $10^{6}$ \\
horizontal scale & $6 H_{p}$ & $\sim 1 H_{p} ?$ \\
boundary layers & photosphere & $?$ \\
\hline \hline
\end{tabular}

While numerical simulations show that photospheric velocity fields consist partly of overshooting convective motions and partly of waves (p-modes), the extrapolation of these results to stellar interiors is questionable, because of the vastly differing time-scales (see Table 1). We know the typical scales of solar granulation rather precisely and it is possible to advance a 3D numerical model with a time step bound to the Courant condition over some thermal time scales.

Our knowledge about the conditions near the bottom of the solar convection zone is much worse: the typical scales and the structure of the boundary are very uncertain. The range of time scales requires different algorithms and much more powerful computers. To reduce the computational demand for a model of very deep convection one is forced to introduce some simplifying scaling into the numerical models. The construction of proper boundary conditions (e.g. to mimic the possible influence of the small-scale surface granulation on the very deep layers) is a major problem.

There are several candidates as mixing mechanisms acting near the boundary of a convection zone in the stellar interior. The classical ballistic picture has been refined by Zahn (1991). The conditions in the stellar interior cause the boundaries of a convection zone to act almost as rigid walls. Convective elements might "smash" against these walls and excite gravity waves in the stable medium which then mix the material. Rotation has to be taken into account (Brun, these proceedings) and even magnetic phenomena might play a role (Charbonneau, these proceedings). 
If the computational box of a simulation includes convectively stable buffer regions, the turnover flow extends well beyond the convection zone into these stable layers. The transition region from unstable to stable conditions is complex. Nevertheless, in simulations of shallow convection zones in DA white dwarfs and A-type main-sequence stars (Freytag et al. 1996) the velocity amplitude shows a simple exponential decline with distance from the convection zone. The diffusion coefficent due to overshoot behaves like $D_{\text {over }}(z)=t_{\text {char }} \cdot V_{\text {rms }}^{2}(z)$, where $t_{\text {char }}$ is a characteristic dynamical time scale, $V_{\text {rms }}$ the rms value of the vertical velocity, and $z$ the distance from the unstable zone. A detailed description of the velocity field in the transition region is not necessary, because there (and within the convection zone) the convective mixing occurs practically instantaneously.

The simulations give values for $t_{\text {char }}$ and $V_{\text {rms }}$ only for some shallow surface convection zones. To apply the results to stellar interiors, one has to assume that the basic mechanism still is the same and that typical velocities can be used from mixing-length theory. The scale height of the diffusion coefficient has to be treated as free parameter and needs calibration to the conditions at hand. This description of overshoot has been successfully applied by Herwig et al. (1997) to evolutionary models of AGB stars. But Schlattl \& Weiss (1999) find that for the Sun a turbulent diffusion approach is to be preferred.

The existing radiation hydrodynamics simulations demonstrate that there are several causes for convectively induced mixing beyond a convection zone: e.g. by the velocities of the overturning motions, by acoustic and by gravity waves. Radiation hydrodynamics codes are useful tools in the investigation of mixing mechanisms in stars. However, the algorithms have to be adapted to the peculiar conditions in the stellar interior and it is a big challenge to set up a simulation with parameters appropriate for this extreme regime.

\section{References}

Asplund, M., Ludwig, H.-G., Nordlund, $\AA$, Stein, R.F. 2000, A\&A, 359, 669

Christensen-Dalsgaard, J., Monteiro, M.J.P.F.G., Thompson, M.J. 1995, MNRAS, 276, 283

Freytag, B., Ludwig, H.-G., Steffen, M. 1996, A\&A, 313, 497

Herwig, F., Blöcker, T., Schönberner, D., El Eid, M. 1997, A\&A, 324, L81

Krieg, J., Kneer, F., Koschinsky, M., Ritter, C. 2000, A\&A, 360, 1157

Kupka, F. 1999, ApJ, 526, L45

Landstreet, J.D. 1998, A\&A, 338, 1041

Pols, O.R., Tout, C.A., Schröder, K.-P., Eggleton, P.P., Manners, J. 1997, MNRAS, 289, 869

Schlattl, H., Weiss, A. 1999, A\&A, 347, 272

Stein, R.F., Nordlund, $\AA$ 1998, ApJ, 499, 914

Zahn, J.-P. 1991, A\&A, 252, 179 